\title{
Different hierarchy of avalanches observed in the Bak-Sneppen evolution model
}

\author{
W. $\mathrm{Li}^{1, *}$ and X. Cai ${ }^{1,2,3, \dagger}$ \\ ${ }^{1}$ Institute of Particle Physics, Hua-zhong Normal University, Wuhan 430079, China \\ ${ }^{2}$ INP, Université de Paris-Sud and CNRS-IN2P3, F-91406 Orsay Cedex, France \\ ${ }^{3}$ CERN, CH-1211, Geneva 23, Switzerland
}

(Received 18 June 1999; revised manuscript received 3 September 1999)

\begin{abstract}
A quantity $\bar{f}$ denoting the average fitness of an ecosystem is introduced in the Bak-Sneppen model. Through this quantity, a different hierarchy of avalanches, $\bar{f}_{0}$ avalanche, is observed in the evolution of Bak-Sneppen model. An exact gap equation, governing the self-organization of the model, is presented in terms of $\bar{f}$. It is found that self-organized threshold $\bar{f}_{c}$ can be exactly obtained. Two basic exponents of the new avalanche $\tau$, avalanche distribution, and $D$, avalanche dimension are given through simulations of one- and two-dimensional Bak-Sneppen models. It is suggested that $\bar{f}$ may be a good quantity in determining the emergence of criticality.

PACS number(s): 87.10.+e, 05.40. $-\mathrm{a}, 05.65 .+\mathrm{b}$
\end{abstract}

The term avalanche may originate from the phenomena which occur in nature. It is referred to as sequential events which may cause devastating catastrophes. The phenomena of avalanches are ubiquitous in nature. The canonical example of the avalanche is the mountain slide, during which a great mass of snow and ice at a high altitude slide down a mountain side, often carrying with it thousands of tons of rock, and sometimes destroying forests, houses, etc., in its path [1]. Since avalanches occur everywhere, from the rice pile, to the Himalayan sand piles; from the river network, to the earthquake, starquakes, and even solar flares; from the biology to the economy [2], etc., it is hence proposed [2] that avalanches may be the underlying mechanism of the formation of various geographical structures and complex organisms, e.g., brains, etc. (It is now even proposed by Meng et al. [3] that the formation of colorless gluon clusters may be attributed to avalanches intrigued by emission or absorption of gluons.) From this point of view, avalanches can be viewed as the immediate results of complex systems, and hence can be used as the theoretical justification for catastrophism. This is because if the real world is complex then the catastrophes are inevitable and unavoidable.

Plenty of patterns provided by nature exhibit coherent macroscopic structures developed at various scales and do not exhibit elementary interconnections. They immediately suggest seeking a compact description of the spatiotemporal dynamics based on the relationship among macroscopic elements rather than lingering on their inner structure [4]. That is, one needs to condense information when dealing with complex systems. Maybe only this way is efficient and turns out successful.

As known, the avalanche is a kind of macroscopic phenomenon driven by local interactions. The size of the avalanche, spatial and temporal as well, may be sensitive to the initial configuration, or more generally, the detailed dynamics of the system. However, the distribution of avalanches,

\footnotetext{
*Electronic address: liw@iopp.ccnu.edu.cn

${ }^{\dagger}$ Electronic address: xcai@wuhan.cngb.com
}

i.e., Gutenberg-Ritcher law [5], or equivalently, power law, does not depend on such details due to the universality of complexity. Hence, in this sense an avalanche study may be an appropriate tool in studying various complex phenomena. On the other hand, observation of a great variety of patterns, such as self-similar, fractal behavior in nature [6-9], $1 / f$ noise in quasar [10], river flow [11], and brain activity [12], and many natural and social phenomena, including earthquakes, economic activity, and biological evolution suggests that these phenomena are signatures of spatiotemporal complexity and can be related via scaling relations to the fractal properties of the avalanches [13]. This suggestion means that the occurrence of these general, empirical phenomena may be attributed to the same underlying avalanche dynamics. Thus, one can see that study of the avalanche is crucial in investigating the critical features of complex systems. It can even be inferred that avalanche dynamics provides much useful information for us to understand the general features of the ubiquitous complexity around us.

Despite the fact that avalanche may provide insight into complexity, the definition of which can be vastly different for various systems, and the same sorts of systems, even the same system. Let us recall some definitions of avalanche given before. In the sand pile model [2], an avalanche is intrigued by adding a grain or several grains of sand into the system at some time and causing the topple of some sites, which may later on cause some other sites to topple. The avalanche is considered over when the heights of all the sites are less than the critical value, say, 4. In the Bak-Sneppen model [14], several kinds of avalanches [13] are presented. For instance, $f_{0}$ avalanche, $G(s)$ avalanche, forward avalanche, backward avalanche, etc. Though these kinds of definitions of avalanche may show various hierarchical structures they manifest the same underlying fractal feature of the system, i.e., self-organized criticality (SOC). Relating all these kinds of avalanches one can provide a general definition of the avalanche for Bak-Sneppen model: An avalanche corresponds to sequential mutations below a certain threshold. One can see that this kind of definition can ensure the mutation events within a single avalanche are casually and 
spatially connected. In addition, with this definition there exists a hierarchy of avalanches, each defined by their respective threshold. It is the hierarchical structure of the avalanche, which exhibits the fractal geometry of the system and implies complexity.

It can be inferred from the definition of an avalanche that there always exists a triggering event which initiates an avalanche and whose effect, that is, causing the avalanche to spread later on within the system, will disappear at the end of the avalanche. The observation of the avalanche through the triggering event, up to now, has been based on the individual level, despite the fact that the avalanche is a macroscopic and global phenomenon of the system studied, in the laboratory, and in nature as well. In the sand pile model, the triggering event is adding a grain or several grains of sand to some site, causing them to topple. In the Bak-Sneppen model, the corresponding triggering event of an avalanche is mutation of the extremal species causing the fitness [14] of the extremal site at the next time step less than a certain threshold. In the above two models triggering events are directly connected with the feature of individuals, e.g., the height of the site in the former or, the fitness of the extremal site in the latter. It can be readily learned that the triggering events, whether in the laboratory or in nature, are not directly related to the global feature of the systems although the avalanche can span the whole system. Generally speaking, the behavior of avalanches is observed through the features of individuals, instead of those of the whole system. However, general features of the complex system may provide insight into knowing the tendency of the evolution of the system. Specifically, global features of a complex system may enable one to understand the critical behavior of the system. It implies that some characteristic quantity, representing the corresponding global features, can be employed in describing complex systems. Furthermore, these quantities ought to be related to avalanche dynamics, and hence can be used to describe complexity emerged in a variety of complex systems. Our aim is to search for or define such quantities and we expect to observe new types of avalanches based on these quantities. Indeed, we obtain a quantity which can be used to define a different hierarchy of avalanches in the Bak-Sneppen model. We suggest that this quantity may be used as a criterion in determining the emergence of criticality. It will be shown later that this type of avalanche still exhibits spatiotemporal complexity in another context.

The Bak-Sneppen model [14] is a very simple evolution model of biology. Despite the simplicity of the model itself, it can exhibit the skeleton of species evolution-punctuated equilibrium. In Bak-Sneppen model, each species is represented by a single fitness. The fitness may represent population of a whole species or living capability of the species [15]. It is a vital quantity and the only one describing the model. No other additional quantities are employed in this oversimplified model. Thus, the fitness is the most important feature of species, and absolutely the most important of the model. So, when considering global feature of the ecosystem, one has to relate it to that of individuals, i.e., fitness.

In this "toy" model (Bak-Sneppen model), random numbers $f_{i}$ chosen from a flat distribution $p(f)$ are assigned independently to each species located on a $d$-dimensional lattice of linear size $L$. At each time step, the extremal site, i.e., the species with the smallest random number, and its $2 d$ nearest neighboring sites, are assigned $2 d+1$ new random numbers also chosen from $p(f)$. This updating process continues indefinitely. After a long transient process the system reaches a statistically stationary state where the density of random numbers in the system vanishes for $f<f_{c}$ and is uniform above $f_{c}$ (the self-organized threshold).

Introduce a new quantity for Bak-Sneppen model. Define the average fitness, denoted by $\bar{f}$, as

$$
\bar{f}=\frac{1}{L^{d}} \sum_{i=1}^{L^{d}} f_{i},
$$

where $f_{i}$ is the fitness of the $i$ th species. Here, we refer to $\bar{f}$ as the average fitness and a global quantity of the system. $\bar{f}$ may represent average population or average living capability of the whole ecosystem. Large $\bar{f}$, i.e., high average fitness, may imply the total population of the system is immense or its average living capability is great, and vice versa. An initial value of $\bar{f}$, denoted by $\bar{f}(0)$, can be easily obtained. As known, at the beginning of the evolution $f_{i}$ 's are uniformly distributed between $(0,1)$. Hence, for an infinitesize system, $\bar{f}(0)$ equals 0.5 . However, for a finite-size system $\bar{f}(0)$ fluctuates slightly due to the finite size effect. It should be pointed out that $\bar{f}(0)$ does not reflect the correlation between species. As the evolution goes on such a correlation tends to be more distinctive. Denote $\bar{f}(s)$ the average fitness of the system at time step $s$ in the evolution. In the $s$ limit, i.e., $s \gg L^{d}, \bar{f}(s)$ may partly reflect information about correlation. As a global quantity, $\bar{f}(s)$ should include information concerning the interaction between species. Hence, it is natural to expect that $\bar{f}$ may be a good quantity in describing the feature of the system as a whole.

Before introducing the different hierarchy of avalanches it is necessary and worthwhile to investigate features of the new quantity $\bar{f}(s)$. First, let us present some theoretical analysis. Recalling the definition of $\bar{f}$ one can see that $\Delta \bar{f}(s)=\bar{f}(s+1)-\bar{f}(s)$ approaches zero in the $L \rightarrow \infty$ limit. An observer can hardly perceive the change in $\bar{f}(s)$ during such a short time period since it is vanishingly small. However, changes at each time step are accumulated to form a relatively distinctive change after a long time, which is perceivable for the observer. This long time period is required to be much larger than the system size, i.e., $s \gg L^{d}$. In other words, $\bar{f}\left(s+s_{0}\right)-\bar{f}\left(s_{0}\right)$ may only be "noticed" when $s$ $\gg L^{d}$ ( $s_{0}$ denotes any initial time step). The variation of $\bar{f}(s)$ is small between two successive time steps, which differs from that of fitness of extremal site. The latter can be very large, say, 1. It should also be expected that there exists an increasing tendency of $\bar{f}(s)$ versus time $s$. This is because at each time step the least fitness is eliminated from the system so the general fitness of the whole system will tend to increase. And due to the slow fluctuation of $\bar{f}(s)$ the increasing in $\bar{f}(s)$ behaves similar to a staircase, i.e., Devil's staircase [2]. Hence, one may expect to observe such behavior, i.e., 


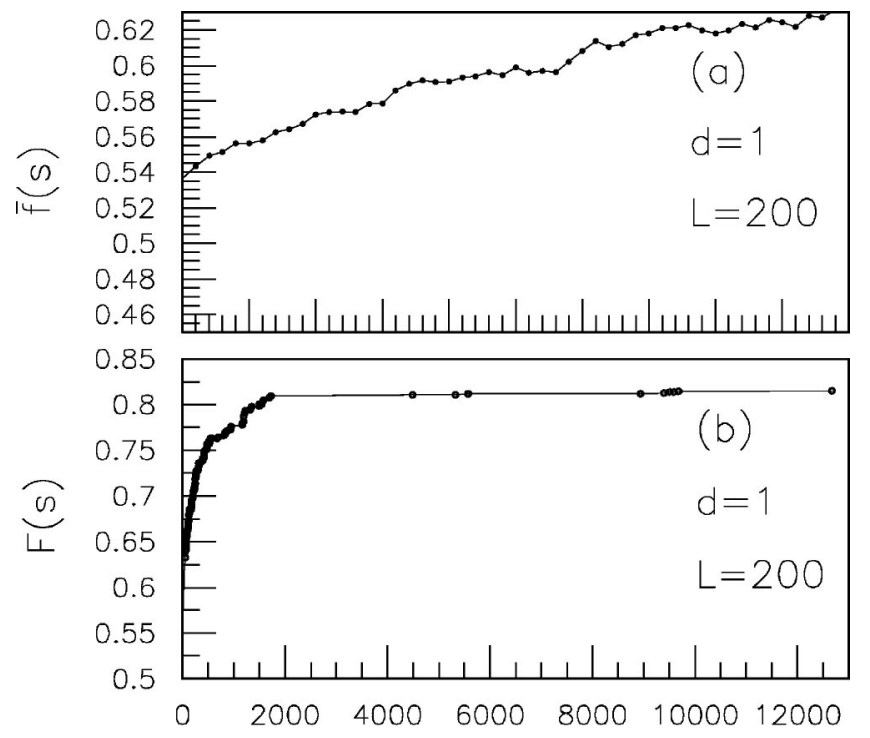

$\mathrm{S}$

FIG. 1. (a) The variation of $\bar{f}$ versus time during a time period for a one-dimensional Bak-Sneppen model of size $L=200$. This shows the hierarchical structure of $\bar{f}$. (b) Punctuated equilibrium of $\bar{f}$ for a one-dimensional Bak-Sneppen model of size $L=200$. We track the increasing signal of $\bar{f}(s)$, i.e., $F(s)$.

punctuated equilibrium [14], of $\bar{f}(s)$ in the evolution of the Bak-Sneppen model.

In order to show the feature of $\bar{f}(s)$ versus time $s$ we perform simulations of the Bak-Sneppen model. At each time step, in addition to the updating of the extremal sites, we also track the signals $\bar{f}(s)$. Figure 1(a) presents the evolution of $\bar{f}(s)$ versus time $s$ during some time period. This plot shows that $\bar{f}(s)$ varies slightly between two successive time steps but tends to increase in the long evolution process.

Introducing another quantity $F(s)$, the gap of the average fitness. The definition of $F(s)$ is given as follows: Initial value of $F(s)$ is equal to $\bar{f}(0)$. After $s$ updates, a large $F(s)>F(0)$ opens up. The current gap $F(s)$ is the maximum of all $F\left(s^{\prime}\right)$, for all $0 \leqslant s^{\prime}<s$. Figure 1(b) shows $F(s)$ as a staircase increasing function of $s$ during the transient. Actually, the gap is an envelope function that tracks the increasing peaks in $\bar{f}(s)$. Indeed, punctuated equilibrium behavior appears in terms of $\bar{f}(s)$.

By definition [14], the separate instances when the gap $F(s)$ jumps to its next higher value are separated by avalanches. Avalanches correspond to plateaus in $F(s)$ during which $\bar{f}(s)<F(s)$. A new avalanche is initiated each time the gap jumps and ends up when the gap jumps again. As the gap increases, the probability for the average fitness $\bar{f}(s)$, to fall below the gap increases also, and larger and larger avalanches typically occur.

We can derive an exact gap equation of $F(s)$, similar to that found in Ref. [16]. Suppose in the system the current gap is $F(s)$. If $F(s)$ is to be increased by $\Delta F$, i.e., from $F(s)$ to $F(s)+\Delta F$, the average number of avalanches needed is $N_{\mathrm{av}}=\Delta F L^{d} /\left[\bar{f}_{c}-F(s)\right]$, where $\bar{f}_{c}$ is the critical value of $\bar{f}(s)$. We can guarantee $N_{\mathrm{av}} \gg 1$ by selecting $\Delta F \gg L^{-d}$. In the large $L$ limit, $N_{\text {av }}$ can be arbitrarily large. Hence, in this limit, the average number of time steps required to increase the gap from $F(s)$ to $F(s)+\Delta F$ is given by the interval $\Delta s=\langle S\rangle_{F(s)} N_{\mathrm{av}}=\langle S\rangle_{F(s)} \Delta F L^{d} /\left[\bar{f}_{c}-F(s)\right]$, where $\langle S\rangle_{F(s)}$ is the average size of avalanche of the plateaus in the gap function. From the law of large numbers the fluctuation of this interval around its average value vanishes. In the $\Delta F \rightarrow 0$ limit, $\Delta s \rightarrow 0$. Taking the continuum limit we can obtain the differential equation for $F(s)$,

$$
\frac{d F(s)}{d s}=\frac{\bar{f}_{c}-F(s)}{L^{d}\langle S\rangle_{F(s)}} .
$$

Note that this equation is exact.

All SOC models, e.g., the BTW sand pile model [17], the earthquake models [18], or Bak-Sneppen model [14], exhibit self-organized criticality in terms of a power-law distribution of the avalanche. It is natural to expect that we can observe SOC in terms of the hierarchical structure of $\bar{f}(s)$, which itself manifests complexity. It is simply another way to observe the same phenomenon by using such a quantity to define the avalanche, which can be observed in different ways. As known, the emergence of complexity is independent of the tools used to observe them provided that these tools are efficient and strong enough. Similar to those used in Refs. $[13,19]$, we present the definition of the $\bar{f}_{0}$ avalanche, where $\bar{f}_{0}\left(0.5<\bar{f}_{0}<1.0\right)$ is only a parameter used to define the avalanche. Suppose at time step $s_{1}, \bar{f}\left(s_{1}\right)$ is larger than $\bar{f}_{0}$. If, at time step $s_{1}+1, \bar{f}\left(s_{1}+1\right)$ is less than $\bar{f}_{0}$, this initiates a creation-annihilation branching process. The avalanche still continues at time step $s^{\prime}$, if all the $\bar{f}(s)$ are less than $\bar{f}_{0}$ for $1 \leqslant s \leqslant s^{\prime}-1$. The avalanche stops, say, at time step $s_{1}+S$, when $\bar{f}\left(s_{1}+S\right)>\bar{f}\left(s_{1}\right)$. In terms of this definition, the size of the avalanche is the number of time steps between subsequent punctuation of the barrier $\bar{f}_{0}$ by the signal $\bar{f}(s)$. In the above example, the size of the avalanche is $S$. It can be clearly seen from Fig. 1(a) that this definition guarantees the hierarchical structure of avalanches-larger avalanches consist of smaller avalanches. As $\bar{f}_{0}$ is lowered, bigger avalanches are subdivided into smaller ones. Hence, the statistics of the $\bar{f}_{0}$ avalanche will inevitably have a cutoff if $\bar{f}_{0}$ is not chosen to be $\bar{f}_{c}$. We can also define a $\bar{f}_{c}$ avalanche. Nevertheless, the $\bar{f}_{0}$ avalanche in the stationary state has the same scaling behavior as the $\bar{f}_{c}$ avalanche provided $\bar{f}_{0}$ close to $\bar{f}_{c}$. We measure the $\bar{f}_{0}$ avalanche distribution for onedimensional (1D) and two-dimensional (2D) Bak-Sneppen models. The simulation results are given in Fig. 2. The exponent $\tau$, defined by $P(S) \sim S^{-\tau}$, is 1.800 for $1 \mathrm{D}$ model and 1.725 for the 2D model. Another exponent $D$, avalanche dimension [13], defined by $n_{\text {cov }} \sim S^{D / d}$, where $n_{\text {cov }}$ is the number of sites covered by an avalanche, and $d$ is the space dimension, is measured. We find $D=2.45$ for the $1 \mathrm{D}$ model and 3.10 for the $2 \mathrm{D}$ model.

Up to now, a question is still unsolved. It is the critical value of $\bar{f}, \bar{f}_{c}$. This may be troublesome if the system size is finite, but when we consider the large $L$ limit, it can be easily accomplished. Recall the evolution of Bak-Sneppen model, 


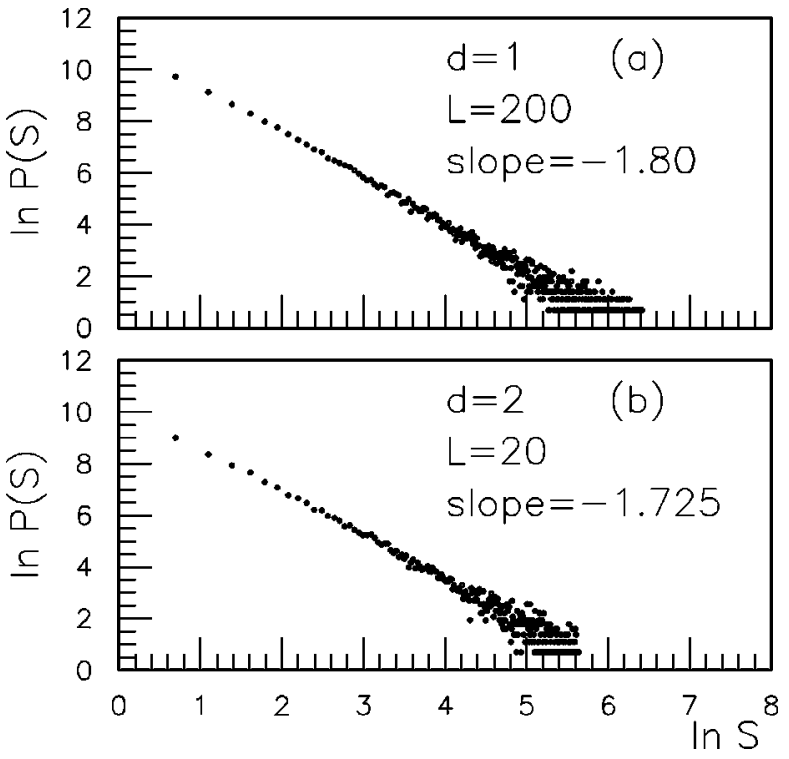

FIG. 2. Distributions of the $\bar{f}_{0}$ avalanche for (a) onedimensional Bak-Sneppen model with size $L=200, \bar{f}_{0}=0.821$ and slope $=-1.800$ and (b) two-dimensional Bak-Sneppen model with size $L=20, \bar{f}_{0}=0.648$ and slope $=-1.725$.

or the detailed research of the model of Ref. [13], the densities of sites with random numbers is uniform above $G$ and vanishes below $G$ when $L \rightarrow \infty$, where $G$ is the gap of extremal site. One can readily obtain

$$
\lim _{L \rightarrow \infty} \bar{F}(s)=\lim _{L \rightarrow \infty} \frac{1+G(s)}{2}
$$

From Eq. (3) one can immediately obtain

$$
\lim _{L \rightarrow \infty} \bar{f}_{c}=\lim _{L \rightarrow \infty} \frac{1+f_{c}}{2} .
$$

Hence, $\bar{f}_{c}$ can be easily determined from Eq. (4). Using the results of $f_{c}$ provided by Refs. $[13,20]$, one can obtain $\bar{f}_{c}$, 0.83351 for the $1 \mathrm{D}$ model and 0.66443 for the 2D model. However, Eqs. (3) and (4) are not valid for a finite-size system, since one cannot ensure the distribution of random numbers during a finite-size system is really uniform. Due to the fluctuation of $\bar{f}(s)$ it is extremely difficult to determine the exact critical value of $\bar{f}$ for a finite-size system. One may estimate $\bar{f}_{c}$ for a finite-size system using the simulation. We find that this value weakly depends on the system size when it is enough large and $\bar{f}_{c}$ will approach the corresponding value for infinite systems. Figure 3 shows the fluctuation of $\bar{f}$ for a $1 D$ model of size $L=200$ near the critical state. We note, in this curve, $\bar{f}$ fluctuates slightly around some average value and does not tend to increase any more during a long time period. We may say that the system approaches its stationary state. In this sense, we suggest that $\bar{f}$ may be a good quantity in determining the emergence of criticality. That is, the great fluctuation of $f_{\min }$ will not help us to determine when we approach the critical state. We need only know the

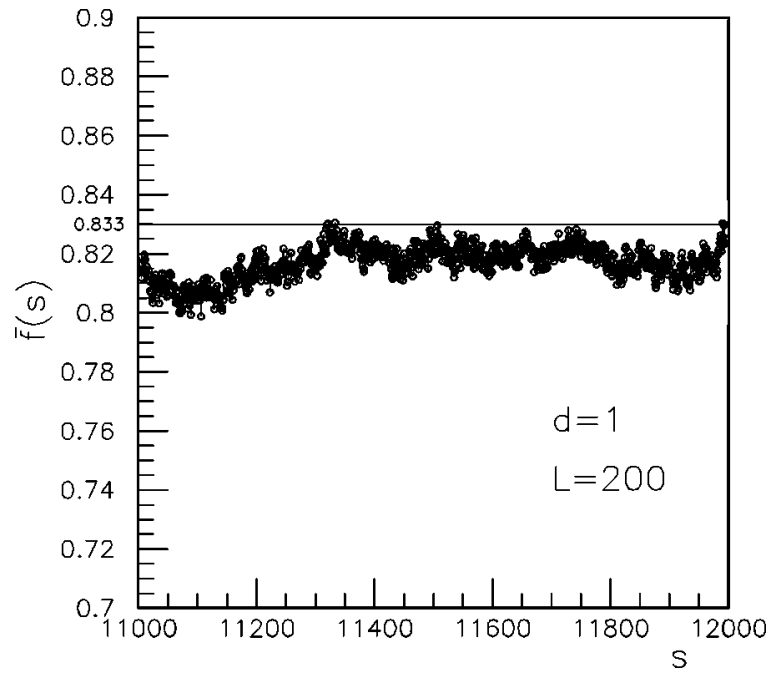

FIG. 3. The fluctuation of $\bar{f}$ around the critical state for a onedimensional Bak-Sneppen model of size $L=200$.

feature of $\bar{f}$. It is more reasonable and easily accepted since $\bar{f}$ is a global quantity and condenses information of the system and its components.

Why do we call the $\bar{f}_{0}$ avalanche a different hierarchy of avalanches? First, this type of avalanche is defined on a global level, in terms of the new global quantity $\bar{f}$. The background of this definition is different than those used before. This type of avalanche reflects the fractal geometry in terms of the global feature. Secondly, one can notice that the exponents $\tau$ of avalanche distribution obtained in our simulations are different than those found in Ref. [13]. From this point of view, one can conclude that this type of avalanche is different than any one observed before.

SOC was suggested by Bak et al. to be the "fingerprints" of a large variety of complex systems, which is represented by a scale-free line on a double logarithm plot. In order to know the criticality of a system one needs to know when the system reaches the stable stationary state where the phase transition occurs. It is extremely difficult and nearly impossible for one to know when a system in nature approaches its critical state. One has to study the ubiquitous fractal geometrical structure carved by avalanches through thousands of millions of years. However, in laboratory experiments and computer simulations, one needs a criterion to determine whether stationary state approaches, even, reaches, since statistics of avalanches may only be done under critical state of the system. Given the Bak-Sneppen model, when the extremal signal $f_{\min }$ approaches the self-organized threshold $f_{c}$ the ecosystem reaches its stationary state. However, $f_{\min }$ itself fluctuates greatly time to time, which brings a big problem in determining the appearance of criticality. Thus, we provide a quantity $\bar{f}$ for a candidate in judging the emergence of criticality. As shown, $\bar{f}$ is relatively stable in a short time period. Hence, when $\bar{f}$ does not tend to increase any more, one may say that the system approaches its stationary state. One can also observe criticality over quite a long time period. 
In conclusion, a different hierarchy of avalanches is observed in the Bak-Sneppen model. A new quantity $\bar{f}$ is presented and suggested by us to be a possible candidate in determining the emergence of criticality. An exact gap equation and simulation results are also given.
This work was supported in part by the NSFC in China and the Hubei NSF. We thank Professor T. Meng for correspondence and helpful discussions. X.C. thanks J. Schukraft and D. Jouan for their hospitality during his visits to CERN and Orsay.
[1] Extracted from Oxford Advanced Learner's Dictionary of Current English with Chinese Translation (Oxford University Press, Hong Kong, 1984).

[2] P. Bak, How Nature Works (Springer-Verlag, New York, 1996).

[3] Meng Ta-chung, R. Rittel, and Zhang Yang, Phys. Rev. Lett. 82, 2044 (1999).

[4] R. Badii and A. Politi, Complexity (Cambridge University Press, London, 1997).

[5] B. Butenberg and C. F. Richter, Ann. Geofis. 9, 1 (1956).

[6] B. B. Mandelbrot, The Fractal Geometry of Nature (Freeman, New York, 1983).

[7] P. H. Coleman, L. Pietronero, and R. H. Sanders, Astron. Astrophys. 200, L32 (1988); L. Pietronero, Physica A 144, 257 (1987).

[8] A. Rinaldo, I. Rodriguez-Iturbe, R. Rigon, E. Ijjasz-Vasquez, and R. L. Bras, Phys. Rev. Lett. 70, 822 (1993).

[9] Fractals in the Earth Sciences, edited by C. C. Barton and P. R. Lapointe (Plenum, New York, 1994).

[10] W. H. Press, Comments Astrophys. 7, 103 (1978).
[11] See, for example, the empirical observations of H. E. Hurst described in Refs. [6] and [8].

[12] F. Gruneis, M. Nakao, and M. Yamamato, Biol. Cybern. 62, 407 (1990).

[13] M. Paczuski, S. Maslov, and P. Bak, Phys. Rev. E 53, 414 (1996).

[14] P. Bak and K. Sneppen, Phys. Rev. Lett. 71, 4083 (1993).

[15] W. Li and X. Cai, cond-mat/9904313.

[16] M. Paczuski, S. Malsov, and P. Bak, Europhys. Lett. 27, 97 (1994).

[17] P. Bak, C. Tang, and K. Wiesenfeld, Phys. Rev. Lett. 59, 381 (1987); Phys. Rev. A 38, 364 (1988); P. Bak and M. Paczuski, Phys. World 6(12), 39 (1993).

[18] P. Bak and C. Tang, J. Geophys. Res. B 94, 15635 (1989); Z. Olami, H. J. S. Feder, and K. Christensen, Phys. Rev. Lett. 68, 1244 (1992); K. Christensen and Z. Olami, Phys. Rev. A 46, 1829 (1992).

[19] M. Paczuski, S. Malsov, and P. Bak, Europhys. Lett. 28, 295 (1994).

[20] P. Grassberger, Phys. Lett. A 200, 277 (1995). 